\title{
Solidification of Syndiotactic Polystyrene by a Continuous Cooling Transformation Approach
}

\author{
V. LA CARRUBBA, S. PICCAROLO, V. BRUCATO \\ Chemical Engineering Department, Università di Palermo, Viale delle Scienze edificio 6, 90128 Palermo, Italy
}

Received 28 March 2007; revised 14 May 2007; accepted 20 May 2007

DOI: 10.1002 / polb.21254

Published online in Wiley InterScience (www.interscience.wiley.com).

\begin{abstract}
Syndiotactic polystyrene (sPS) was solidified from the melt under drastic conditions according to a continuous cooling transformation methodology developed by the authors, which covered a cooling rate range spanning from approximately 0.03 to $3000{ }^{\circ} \mathrm{C} / \mathrm{s}$. The samples produced, structurally homogeneous across both their thickness and surface, were analyzed by macroscopic methods, such as density, wide-angle X-ray diffraction (WAXD), and microhardness (MH) measurements. The density was strictly related to the phase content, as confirmed by WAXD deconvolution. The peculiar behavior encountered (the density first decreasing and then increasing with the cooling rate) was attributed to the singularity of the phases formed in sPS; that is, one of the crystalline phases $(\alpha)$ was less dense than the amorphous phase, and the latter, in turn, was less dense than the other crystalline phase $(\beta)$. With an increasing cooling rate, the thermodynamically stable phase $(\beta)$ disappeared first, and it was followed by the $\alpha$ phase. On the other hand, the MH values remarkably depended on the amount of the $\beta$ phase, the $\alpha$-phase content influencing the mechanical properties only to a minor extent. The behavior of the crystallization kinetics was described through a modified multiphase Kolmogoroff-Avrami-Evans model for nonisothermal crystallization. (C2007 Wiley Periodicals, Inc. J Polym Sci Part B: Polym Phys 45: 2688-2699, 2007
\end{abstract}

Keywords: crystal structures; crystallization; polystyrene; structure-property relations; syndiotactic

\section{INTRODUCTION}

Syndiotactic polystyrene (sPS) is a new-generation polymer characterized by peculiar features, among which the crystallization behavior is worth mentioning, leading to numerous crystalline modifications: $\alpha, \beta, \gamma$, and $\delta$. With respect to conventional amorphous polystyrene, sPS exhibits a comparable glass transition temperature, a melting point, and, above all, remarkable properties at elevated temperatures, such as Young's modulus and dimensional stability. ${ }^{1}$

Correspondence to: V. La Carrubba (E-mail: lacarrubba@ dicpm.unipa.it)

Journal of Polymer Science: Part B: Polymer Physics, Vol. 45, 2688-2699 (2007)

() 2007 Wiley Periodicals, Inc.
In the primary crystalline polymorphs, the $\alpha$ and $\beta$ phases, the backbone of the polymer chain adopts an all-trans planar zigzag (tttt) ${ }_{n}$ conformation, with an identity period of $0.51 \mathrm{~nm}$. The $\beta$ phase, formed during crystallization on cooling from the melt, is characterized by an orthorhombic packing of the chains with a theoretical density of $1.09 \mathrm{~g} \mathrm{~cm}^{-3}$.

Although both phases may form by crystallization from the melt, the $\alpha$ phase is kinetically favored, whereas the $\beta$ phase is thermodynamically more stable. ${ }^{3-6}$ Hence, the crystallization of the $\beta$ form has an activation energy exceeding that of the $\alpha$ form. ${ }^{7}$ It has also been observed that for the $\alpha$ phase, which can also be obtained by cold crystallization from the amorphous phase, a close packing of chains is hindered by 
the phenyl rings, and this results in a hexagonal structure. Because of the loose packing of chains, this phase has a theoretical density of only $1.03 \mathrm{~g} \mathrm{~cm}^{-3}$, which has been reported to be even lower than that of amorphous sPS (1.04 g $\left.\mathrm{cm}^{-3}\right){ }^{8-17}$

Barnes et al. ${ }^{18}$ showed that, as a result of the close resemblance of the densities of the amorphous and crystalline phase, the small-angle Xray scattering pattern hardly shows any contrast below the glass transition temperature. As a result, the density of the amorphous phase is intermediate between the densities of the $\alpha$ and $\beta$ phases (i.e., $\rho_{\alpha}<\rho_{\text {am }}<\rho_{\beta}$ ).

As far as the properties are concerned, the $\beta$ phase exhibits better mechanical properties and solvent resistance than $\alpha$ does. ${ }^{19}$

The remaining crystalline modifications, $\gamma$ and $\delta$, produced by crystallization from solution, exhibit a helicoidal chain conformation with an identity period of $0.77 \mathrm{~nm}^{3}$ Additionally, two mesomorphic phases have been observed, with a helical conformation ${ }^{20,21}$ and with a zigzag conformation. $^{22,23}$

Guerra et al. ${ }^{3}$ showed that its complex polymorphism depends on a combination of concurrent factors, such as the cooling rate, the parent's previous initial crystalline form before melting, the maximum temperature $\left(T_{\max }\right)$, and the residence time in the molten state.

Although the total crystallinity does not depend on $T_{\max }$, the relative amounts of $\alpha$ (kinetically favored) and $\beta$ (thermodynamically more stable) are severely influenced by its value: the lower $T_{\max }$ is, the larger the percentage is of the $\alpha$ phase. ${ }^{1}$ The $\alpha$ phase normally converts into the $\beta$ phase for temperatures close to its thermodynamic melting point. ${ }^{7}$

Differential scanning calorimetry (DSC) results by Li et al. ${ }^{24}$ highlight the dependence of the complex sPS polymorphism on the cooling rate: high cooling rates $\left(>80{ }^{\circ} \mathrm{C} / \mathrm{min}\right)$ lead to the onset of the $\alpha$ phase, whereas low ones $\left(<10{ }^{\circ} \mathrm{C} /\right.$ $\mathrm{min}$ ) give rise to the preferential development of the thermodynamically stable $\beta$ phase, intermediate cooling rates leading to a mixture of $\alpha$ and $\beta$ phases. Similarly, Filho and Vittoria ${ }^{25}$ measured the crystallinity be means of infrared spectroscopy, confirming that the crystalline content decreases with an increasing cooling rate.

On the other hand, Chiu et al. ${ }^{5}$ investigated the effect of $T_{\max }$ in the molten state and the crystallization temperature during isothermal experiments, and they concluded that, with decreasing $T_{\max }$ (the crystallization temperature being constant), the reciprocal crystallization half-time decreases along with the linear crystal growth rate (evaluated with polarized optical microscopy) and the final crystalline content, in line with the behavior commonly encountered in semicrystalline polymers. Chiu et al. ${ }^{6}$ studied also the nonisothermal crystallization behavior, observing that high values of $T_{\max }$ followed by a slow cooling lead to a negligible final $\alpha$-phase content. Similar results were found by Lin et al. $^{26}$ and $\mathrm{Bu}$ et al., ${ }^{27}$ who showed that the $\beta$ form significantly increases, whereas the $\alpha$ form decreases, as the cooling rate decreases.

Crystallization kinetics under isothermal and nonisothermal conditions were studied by Lawrence and Shinozaki ${ }^{28}$ with the aim of determining the kinetic parameters according to Nakamura's approach and relating them to the morphology observed.

As for the influence of pressure, Rastogi ${ }^{29}$ showed that pressure values on the order of 1500 bar determine a transition from a well-ordered crystalline $\alpha$ phase to a more chaotic, amorphous-like phase, the process being irreversibly driven by the density difference between the amorphous and $\alpha$ phases $\left(\rho_{\alpha}<\rho_{\text {am }}\right)$. On the contrary, when an $\alpha$ or $\gamma$ sample is kept at $240{ }^{\circ} \mathrm{C}$ under a moderate pressure $(57$ bar) in the presence of $\mathrm{CO}_{2}$, a transition to the $\beta$ phase normally takes place. ${ }^{30}$

The aim of this work is to investigate the solidification behavior of sPS over a wide range of cooling rates comparable to those typically adopted in industrial processes (from ca. 0.03 to $3000{ }^{\circ} \mathrm{C} / \mathrm{s}$ ) through a continuous cooling transformation (CCT) protocol already described in detail, ${ }^{31}$ in which a thin polymer sample is quenched by a suitable cooling medium while its temperature is recorded. Sample homogeneity is ensured, and an almost flat temperature distribution is attained dynamically during the quench, the developed structure and morphology depending on the thermal history alone. A unique relationship can thus be assessed between the structure and morphology that develop during solidification under quiescent conditions and the applied thermal history. Because the latter can only be recorded and not controlled, the approach is very similar to the one adopted in metallurgy for studying structure development in metals. ${ }^{31}$ The complete thermal history during cooling being recorded, it can be used as input information for integrating a suit- 
able equation for crystallization kinetics. Furthermore, a rough but effective measure of quenching effectiveness can be drawn from the cooling rate at the temperature at which the kinetic constant, $K(T)$, attains the maximum value. An effective relationship with the structure and morphology can thus be built once the independent variable has been identified as the characteristic cooling rate at which the material can crystallize at the maximum speed.

The dependence of the density, microhardness $(\mathrm{MH})$, and crystalline phase content, drawn from wide-angle X-ray diffraction (WAXD) deconvolution, on the cooling rate is highlighted; the results are interpreted in the framework of the Kolmogoroff-Avrami-Evans (KAE) crystallization kinetics space-filling approach. ${ }^{32-35}$

The obtained information significantly extends available observations to solidification conditions usually met in polymer processing. The measurement of the mechanical properties through $\mathrm{MH}$ provides additional means for the interpretation of structure-property relations in this polymer.

\section{EXPERIMENTAL}

sPS with a weight-average molecular weight of $320,000 \mathrm{~g} / \mathrm{mol}$ and a polydispersity index (weight-average molecular weight/number-average molecular weight) of 3.9 was kindly supplied in pellet form by Dow Chemical Co.

Before being quenched, the sample was held between two identical flat metal slabs and heated to a suitably high temperature while flowing in nitrogen. To prevent any memory effect, a temperatures as high as $300{ }^{\circ} \mathrm{C}$ and a permanence time in the melt of 10-15 min were chosen: literature data confirm that under these conditions, any previous memory of the $\alpha$ form is plausibly removed. . $^{1,3,7,36}$

Different thermal histories were achieved by changes in the cooling medium type (water vs air), flow rate, and thickness of the metal sample holder. Once the sample reached the final temperature, it was immediately removed from the sample assembly and kept at a low temperature $\left(-30{ }^{\circ} \mathrm{C}\right)$ before further characterization. ${ }^{31}$

Although a characteristic time constant could be used to identify and quantify the cooling process, ${ }^{37}$ an equivalent parameter was preferred: the average cooling rate in the range of temperatures within which the polymer solidifies. ${ }^{38}$
This parameter indeed involves not only the imposed experimental time constant but also the characteristic range of temperatures in which a given polymer solidifies, which is usually rather narrow (of the order of $10{ }^{\circ} \mathrm{C}$ ). For the polymer examined in this work (sPS), an average cooling rate around $200{ }^{\circ} \mathrm{C}$ was chosen as a parameter characterizing the cooling effectiveness. ${ }^{31}$

After solidification under controlled cooling conditions, standard techniques of structure and morphology bulk characterization were applied, and the sample produced was assumed to be homogeneous. This not only relied on direct observation of the sample by optical microscopy ${ }^{38}$ but was also confirmed through the modeling of the transient heat conduction problem, which, by suitable control of the sample and sample holder thickness and cooling medium physical properties, led to the conclusion that most of the resistance to heat flux was localized at the solid-fluid interface. ${ }^{39}$

Therefore, the solidified samples were analyzed by macroscopic methods: DSC, WAXD, density, and $\mathrm{MH}$.

The density was measured in a density gradient column filled with a mixture of $n$-heptane and carbon tetrachloride and set at a temperature of $15{ }^{\circ} \mathrm{C}$. The measurement resolution was about $0.0005 \mathrm{~g} / \mathrm{cm}^{3}$. A Vickers MHT-10 microindenter from Anton Paar was employed for $\mathrm{MH}$ measurements determined by the well-established procedure. ${ }^{40}$

The X-ray diffraction measurements were performed with a Bruker model D8 Advance diffractometer in the interval of $5-45^{\circ}$, with a $0.05^{\circ}$ resolution and an accumulation time of $2 \mathrm{~s}$ per step. A multiparameter deconvolution technique was used to estimate the phase content from the X-ray patterns. ${ }^{41}$ The procedure allowed the evaluation of the $\alpha, \beta$, and amorphous mass fractions for all the samples solidified at different cooling rates by the simultaneous fitting of suitable peak functions to the respective WAXD patterns. The procedure is particularly suitable for WAXD data with a limited angular range of intensities.

\section{RESULTS AND DISCUSSION}

Figure 1(a) shows the isothermal crystallization detected by DSC: the temperature interval ranges from 240 to $252{ }^{\circ} \mathrm{C}$. The lower limit is determined by the finite transient of the instrument needed to attain a constant temperature 


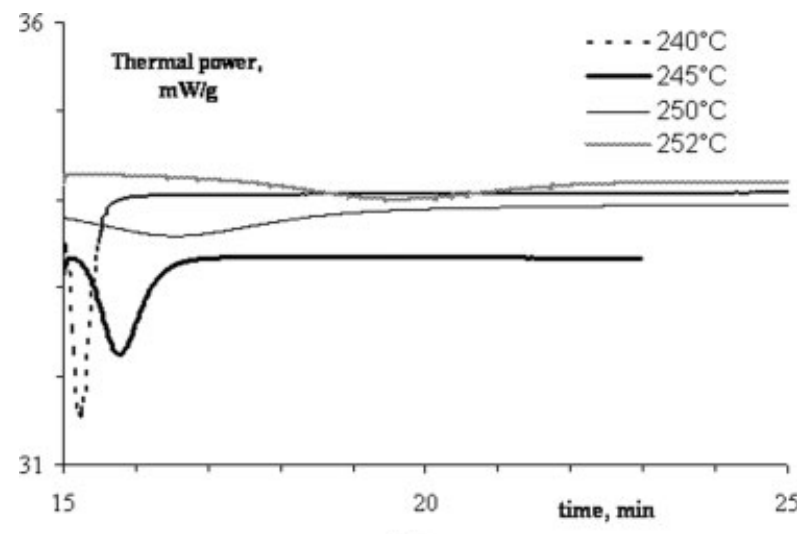

(a)

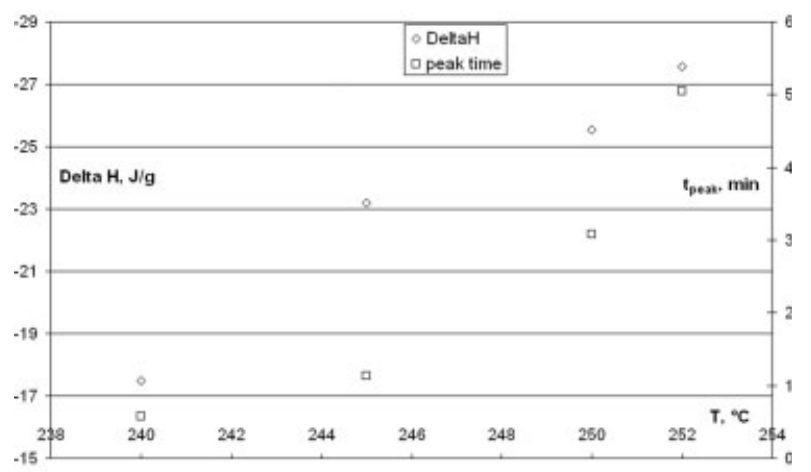

(b)

Figure 1. (a) DSC isothermal crystallization runs and (b) crystallization enthalpy and crystallization half-time as a function of the temperature in isothermal tests.

that is eventually superimposed to the beginning of the crystallization process when it becomes comparable, that is, on the order of 1 min or less for high undercoolings. The upper limit on the other side relates to the slow dynamics of the crystallization process, which leads to a power level of the signal below the instrument sensitivity (ca. $10^{-6} \mathrm{~W}$ ).

Figure 1(b) reports the crystallization enthalpy and half-time as a function of the temperature during isothermal tests. Although it can be shown that the latter follows the usual Arrhenius-like dependence on temperature [the crystallization half-time being the reciprocal of the Avrami crystallization kinetic rate constant $(K)$ ], the former linearly increases with the temperature. The small range of temperatures in which isothermal crystallization can be detected suggests that sPS is a fast crystallizing polymer, comparable to isotactic polypropylene, polybutene-1, and several polyamides, although the large sensitivity of the crystallization enthalpy to the temperature shows that the crystallization kinetics must be slower than the crystallization kinetics of these polymers.

As for the cooling ramps (Fig. 2), the investigated range goes from 0.01 to $2{ }^{\circ} \mathrm{C} / \mathrm{s}$ without loss of control down to apparent temperatures as low as $100{ }^{\circ} \mathrm{C}$, that is, when the crystallization process has been completed. Figure 2 shows that both the onset and peak temperatures as well as the crystallization enthalpy decrease as a function of the cooling rate, although the enthalpy in this case decreases only approximately $15 \%$, whereas in the case of isothermal runs, for a $12{ }^{\circ} \mathrm{C}$ change in the crystallization temperature, the observed change in the crystallization enthalpy is definitely larger.

For the samples melt-solidified at a high cooling rate, insights into the crystallization kinetics can be obtained only indirectly from a postmortem analysis of the developed structure.

Figure 3 presents WAXD patterns of the quenched samples collected in the $2 \theta$ range of $5-45^{\circ}$ but shown in the angular range of $5-19^{\circ}$ to increase readability. Vertical bars mark the positions of the crystalline peaks; those of the $\alpha$ phase are marked by solid lines, and those of the $\beta$ phase are marked by dotted lines. Their positions are summarized in Table $1 .^{3}$ A qualitative analysis of the patterns immediately points out the polymorphic behavior of sPS under rapid cooling conditions: in particular, one first observes that the material is able to crystallize at cooling rates as high as $169{ }^{\circ} \mathrm{C} / \mathrm{s}$, whereas for larger cooling rates (patterns of samples nominally quenched at 667 and $2600{ }^{\circ} \mathrm{C} / \mathrm{s}$ ), the patterns do not show any even broader reflections and can be assumed to describe a completely

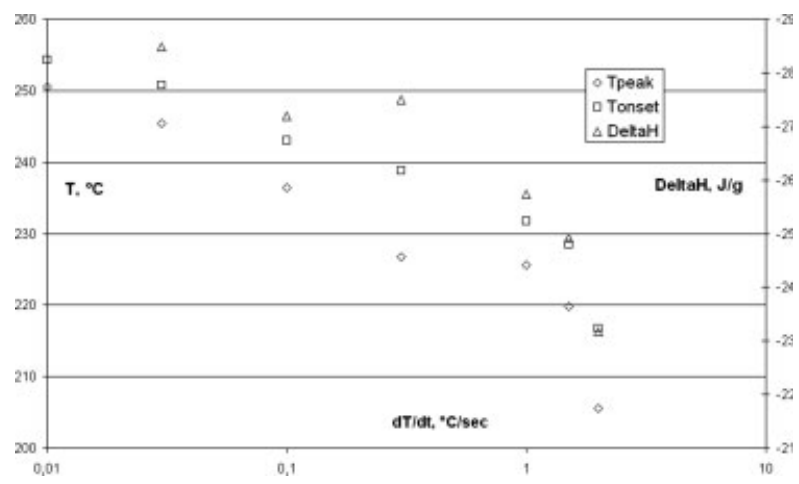

Figure 2. Onset temperature, peak temperature, and crystallization enthalpy as a function of the cooling rate during DSC cooling ramps. 


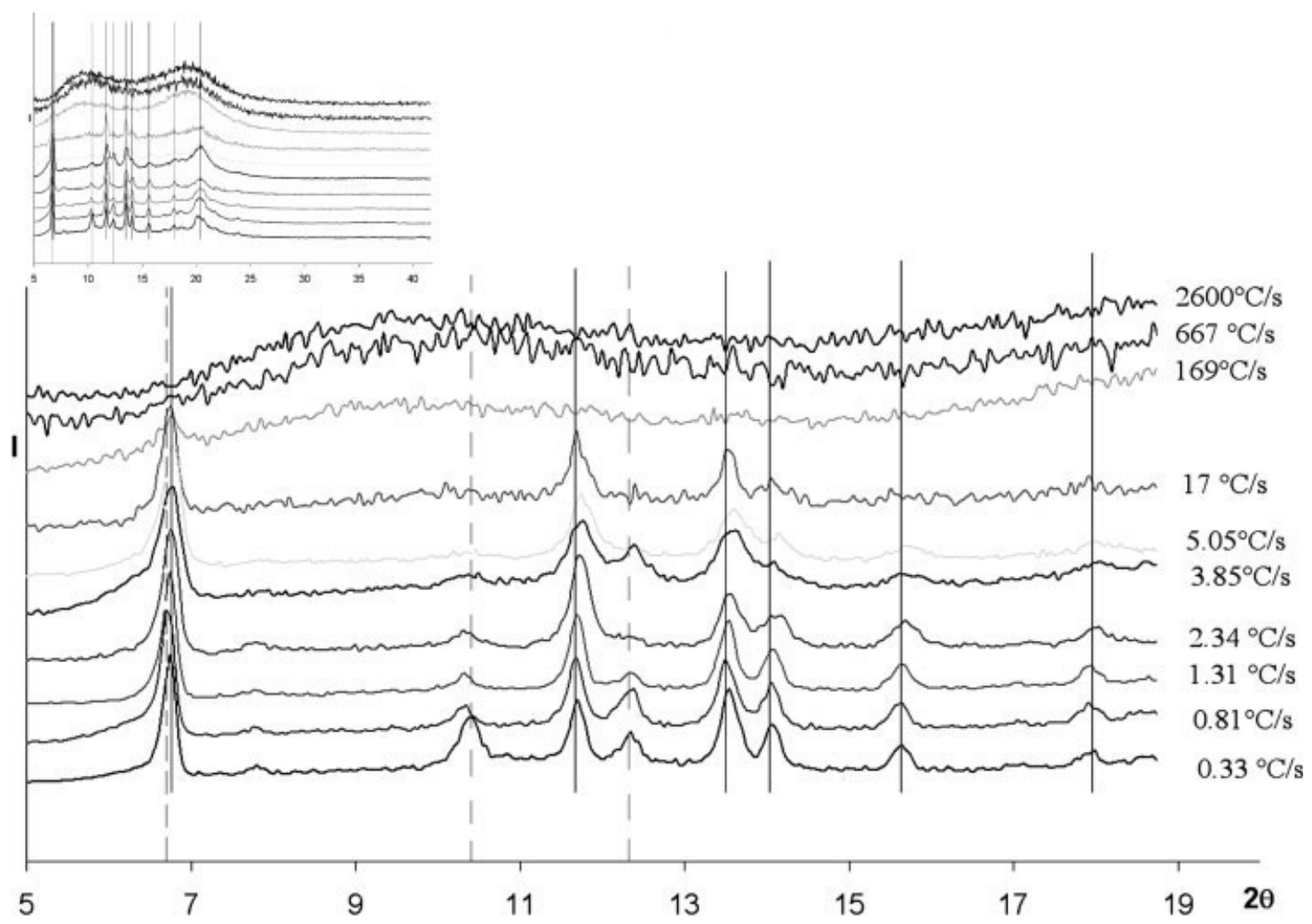

Figure 3. WAXD patterns of sPS samples quenched at different cooling rates (see Table 2). The solid, vertical lines indicate peaks of the $\alpha$ phase; the dotted, vertical lines indicate peaks of the $\beta$ phase.

amorphous material, although one cannot exclude the possibility of some structural order in the disordered state due to the nonequilibrium conditions of the amorphous state. ${ }^{42}$

Among the most crystalline samples, that is, those obtained at lower cooling rates, there are three crystalline peaks clearly distinguishable, corresponding to $2 \theta$ angles of $6.8,11.7$, and $13.5^{\circ}$. The first peak (mentioned in the literature as belonging to the $\alpha$ phase; see Table 1) not only is the most intense but is also the only peak exhibiting diffraction in the sample nominally quenched at $169{ }^{\circ} \mathrm{C} / \mathrm{s}$. The two other intense peaks are also related to the $\alpha$ phase, still remaining evident up to cooling rates as high as $17{ }^{\circ} \mathrm{C} / \mathrm{s}$. Further $\alpha$-phase peaks with lower intensities are located at $2 \theta$ angles of 15.6, 18.0, and $20.2^{\circ}$ (see Table 1).

When we focus on the most crystalline sample, that is, the one solidified at the lowest cooling rate, $0.33{ }^{\circ} \mathrm{C} / \mathrm{s}$, some more crystalline peaks are visibly related to the $\beta$ phase, positioned at $2 \theta$ values corresponding to 6.1 (next to the $6.76^{\circ}$ $\alpha$-phase reflection), 10.4 , and $13.2^{\circ}$, respectively. Although the same peaks are discernible also for larger cooling rate samples, the intensity of the last two peaks tends beyond question to decrease with an increasing cooling rate, so that they turn out be almost invisible for cooling rates larger than $2{ }^{\circ} \mathrm{C} / \mathrm{s}$.

Finally, by looking at the patterns of the amorphous samples (for cooling rates larger

Table 1. Bragg Distances $\left(d_{\mathrm{obs}}\right)$ and Corresponding $2 \theta$ Positions of the $\alpha$-Phase and $\beta$-Phase X-Ray Reflections

\begin{tabular}{|c|c|c|c|c|c|c|c|c|c|c|}
\hline $\begin{array}{c}\alpha \text { Phase } \\
d_{\text {obs }}(\AA)\end{array}$ & 13.11 & 13.07 & 7.58 & 7.51 & 6.56 & 4.94 & 6.31 & 5.67 & 4.37 & 4.34 \\
\hline $2 \theta$ & 6.7 & 6.8 & 11.7 & 11.8 & 13.5 & 18.0 & 14.0 & 15.6 & 20.3 & 20.5 \\
\hline \multicolumn{11}{|l|}{$\beta$ Phase } \\
\hline$d_{\text {obs }}(\AA)$ & 14.37 & 8.48 & 7.18 & 6.52 & 4.78 & 4.39 & 4.18 & & 2.56 & \\
\hline $2 \theta$ & 6.1 & 10.4 & 12.3 & 13.6 & 18.6 & 20.2 & 21.3 & & 35.0 & \\
\hline
\end{tabular}




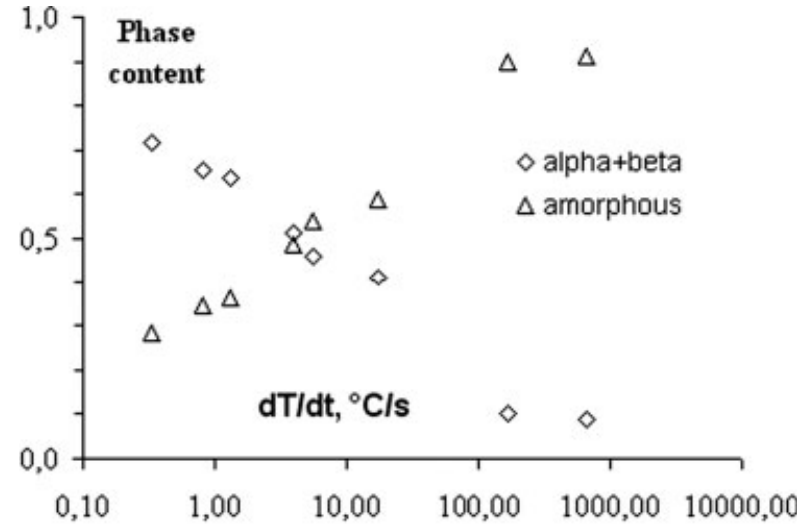

(a)

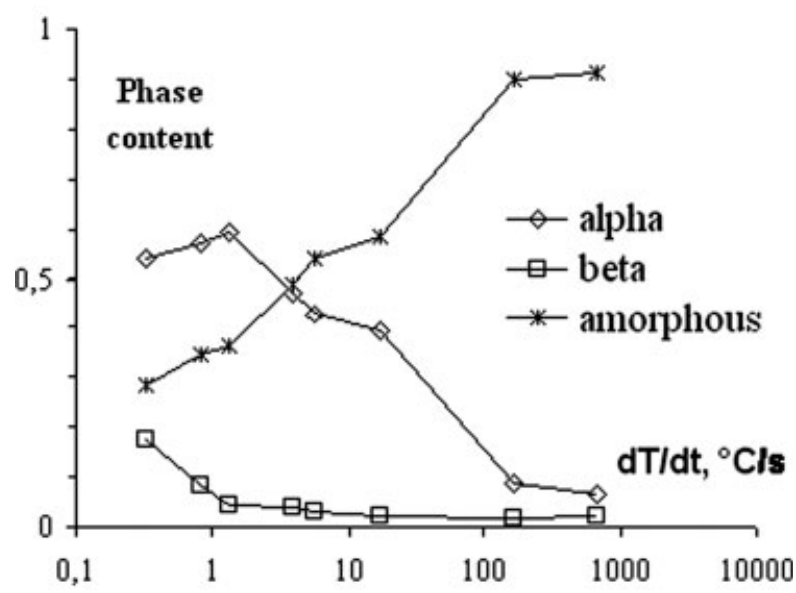

(b)

Figure 4. (a) Overall crystalline $(\alpha+\beta)$ and amorphous fractions as a function of the cooling rate and (b) phase content as a function of the cooling rate.

than $169{ }^{\circ} \mathrm{C} / \mathrm{s}$ ), we can observe only a halo clearly defined by two broad peaks located around $2 \theta$ values of 10 and $19.5^{\circ}$.

In summary, it is evident that all the peaks related to the $\alpha$ crystallinity (the kinetically favored phase) persist up to relatively large cooling rates (of the order of $20{ }^{\circ} \mathrm{C} / \mathrm{s}$ ), whereas the peaks corresponding to the $\beta$-crystalline modification (the thermodynamically favored one) tend to disappear up to relatively low cooling rates (of the order of $2{ }^{\circ} \mathrm{C} / \mathrm{s}$ ).

These qualitative observations find further confirmation in the quantitative results obtained from WAXD pattern deconvolution according to a procedure extensively reported elsewhere.

Figure 4(a) reports the overall crystalline $(\alpha$ $+\beta$ ) and amorphous fractions as drawn from WAXD deconvolution as a function of the cooling rate. An overall crystallinity as high as $70 \%$ is obtained for cooling rates lower than $1{ }^{\circ} \mathrm{C} / \mathrm{s}$, a value rarely observed for commercial polymers if one excludes the case of polyethylene, ${ }^{42}$ whereas at the maximum cooling rates, a residual crystallinity of only a few percent is attained, which is of the same order as the error involved in WAXD deconvolution and therefore is considered negligible. Although the crystallinity measured at low cooling rates in this study sounds unusually high, crystallinity values of the order of $60 \%$ in sPS isothermally crystallized from glass at $150{ }^{\circ} \mathrm{C}$ have been already reported by other authors. ${ }^{36}$

It is more interesting to compare the phase content distributions of the $\alpha$ and $\beta$ phases, as shown in Figure 4(b), where the $\beta$ phase is observed, although in a limited amount, only for low cooling rates $\left(<1{ }^{\circ} \mathrm{C} / \mathrm{s}\right)$, becoming negligible for larger cooling rates. Hence, the $\alpha$-phase content reflects the trend of total crystallinity (see Fig. 4), except for the first three cooling conditions (see Table 2; the samples were solidified at $\left.d T / d t<1{ }^{\circ} \mathrm{C} / \mathrm{s}\right)$.

The phase distribution provides a sound explanation of the density versus the cooling rate, as reported in Figure 5. As a matter of fact, the density dependence on the cooling rate is not monotonous; it shows two branches, first decreasing for low cooling rates and then increasing for high cooling rates, with a minimum value around $1{ }^{\circ} \mathrm{C} / \mathrm{s}$ [see Fig. 5(a)].

The high cooling rate range $\left(d T / d t>500{ }^{\circ} \mathrm{C} / \mathrm{s}\right)$ is characterized by a constant density plateau, as usually happens for all polymers, because drastic cooling conditions normally favor the formation of the amorphous phase or the onset of low-range-order pseudocrystalline phases, highly

Table 2. Solidification Conditions of the Quenched Samples

\begin{tabular}{cc}
\hline Sample & $\begin{array}{c}\text { Nominal Cooling } \\
\text { Rate at } 200{ }^{\circ} \mathrm{C}\left({ }^{\circ} \mathrm{C} / \mathrm{s}\right)\end{array}$ \\
\hline 1 & 0.33 \\
2 & 0.81 \\
3 & 1.31 \\
4 & 2.34 \\
5 & 3.85 \\
6 & 5.05 \\
7 & 17 \\
8 & 169 \\
9 & 667 \\
10 & 2600 \\
\hline
\end{tabular}




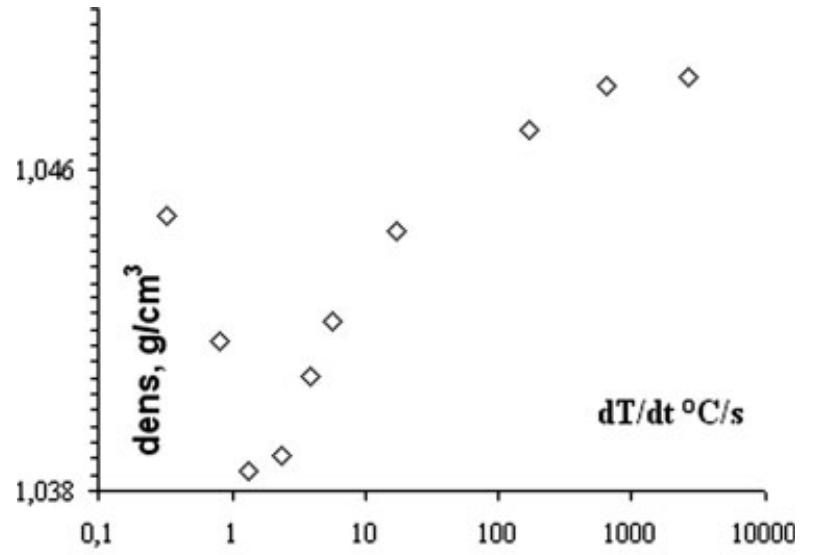

(a)

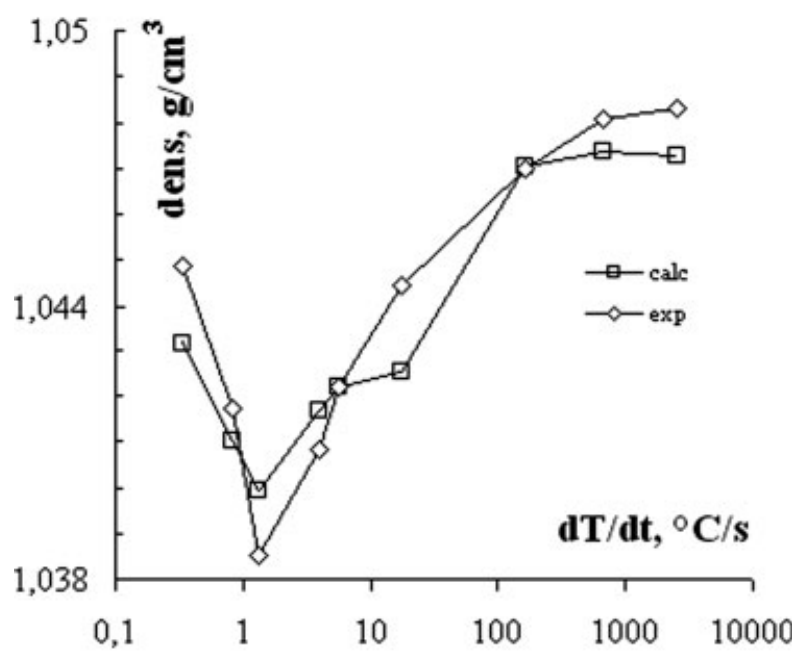

(b)

Figure 5. (a) Density as a function of the cooling rate and (b) experimental and calculated density data.

metastable, which have been identified for a large variety of polymeric materials, including sPS. ${ }^{3,15-17,31,43,44}$ Between 1 and $500{ }^{\circ} \mathrm{C} / \mathrm{s}$, a monotonous density increase can be noticed, in apparent contradiction to the behavior normally observed for semicrystalline polymers, for which a decrease in the density with the cooling rate usually takes place. ${ }^{45}$ However, it should be remembered that the anomalous density value of the amorphous phase (larger than the one of the $\alpha$ phase) is responsible for the observed density increase with an increasing cooling rate because of progressive material amorphization. Finally, in the low cooling rate range, between 0.3 and $1{ }^{\circ} \mathrm{C} / \mathrm{s}$, a continuous monotonous density decrease takes place, with a density minimum for a cooling rate of approximately $1{ }^{\circ} \mathrm{C} / \mathrm{s}$.
To understand this behavior, one must remember that at low cooling rates, the thermodynamically most stable phase is $\beta$, whereas at larger cooling rates, only the kinetically favored one is detected $(\alpha)$. Hence, the observed density minimum results from a competition between the thermodynamically stable $\beta$ phase (the densest) forming for low cooling rates and the kinetically favored $\alpha$ phase (the least dense) forming for moderate cooling rates.

On the basis of this experimental evidence, the minimum in the density data presented in Figure 5(a) can be easily understood. If volume conservation is assumed, the overall density $(\rho)$ can be obtained as follows:

$$
\frac{1}{\rho}=\frac{X_{\alpha}}{\rho_{\alpha}}+\frac{X_{\beta}}{\rho_{\beta}}+\frac{X_{\mathrm{am}}}{\rho_{\mathrm{am}}}
$$

The phase densities $\left(\rho_{\alpha}, \rho_{\beta}\right.$, and $\left.\rho_{\text {am }}\right)$ can be obtained from the literature, ${ }^{1}$ and the phase fractions $\left(X_{\alpha}, X_{\beta}\right.$, and $\left.X_{\mathrm{am}}\right)$ are drawn from WAXD deconvolution [Fig. 4(b)].

Figure 5(b) shows a comparison between the density calculated through eq 1 and the experimental data [shown in Fig. 5(a)]; it is thus possible to accurately predict the density minimum for a cooling rate around $1{ }^{\circ} \mathrm{C} / \mathrm{s}$.

Going back to the latent heat of crystallization and to the onset and peak crystallization temperatures reported in Figure 2, one might speculate that the more pronounced decrease of the crystallization temperature around a cooling rate of $1{ }^{\circ} \mathrm{C} / \mathrm{s}$ might be related to the disappearance of the $\beta$ phase, as already pointed out by several authors, ${ }^{36}$ although the evidence is weak here, the $\beta$-phase fraction being low. For comparison, we report that the crystallization temperature of indium standards, as recorded in cooling ramps from 20 to $100{ }^{\circ} \mathrm{C} / \mathrm{min}$, exhibits a sensitivity to cooling rates as low as $2{ }^{\circ} \mathrm{C}$, thus confirming that the results presented here are not merely associated with instrumental time lags upon increasing cooling rates.

As for the mechanical properties, $\mathrm{MH}$ measurements performed on the same samples led to the results presented in Figure 6. In contrast to the peculiar cooling rate dependence of the density, $\mathrm{MH}$ monotonously decreases as a function of the cooling rate, as is common for all the polymers investigated so far ${ }^{4,45}$ [cf. Fig. 5(a) with Fig. 6(a)]. It is worth noticing, however, that the overall span of $\mathrm{MH}$ from the maximum, 


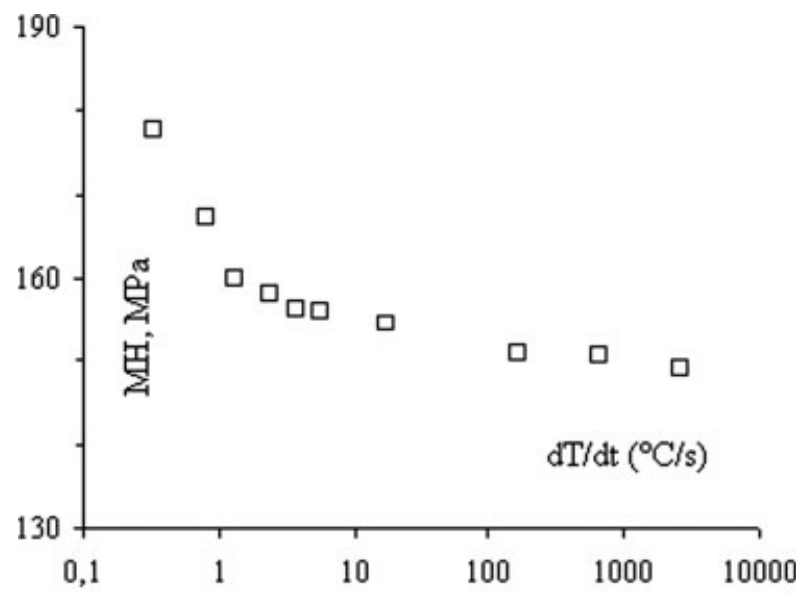

(a)

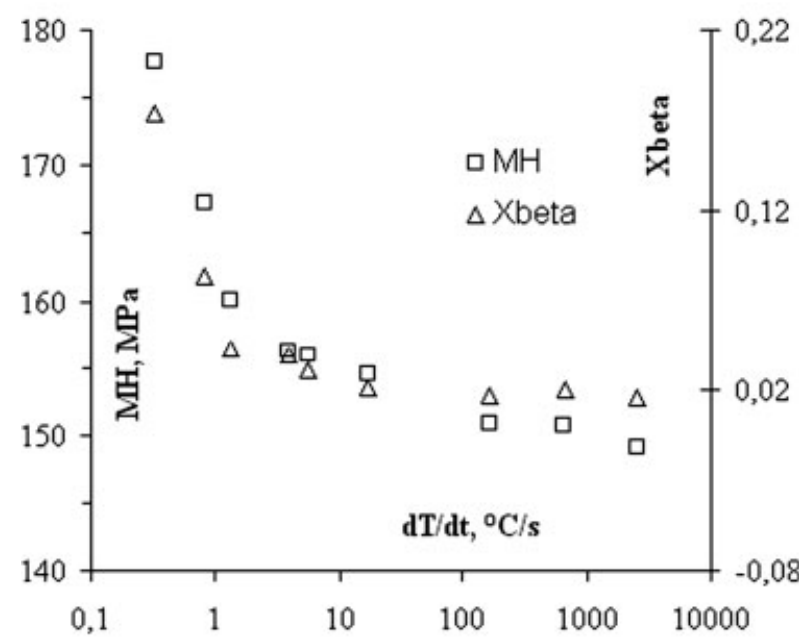

(b)

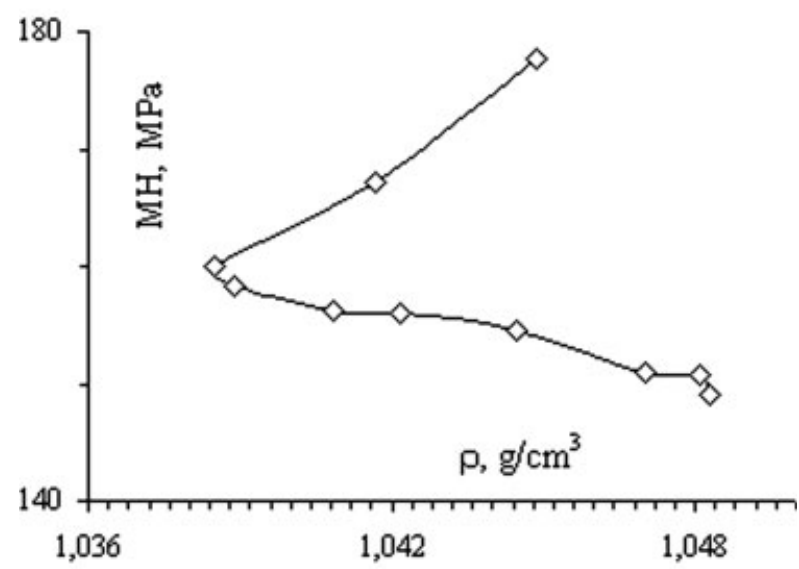

(c)

Figure 6. (a) $\mathrm{MH}$ as a function of the cooling rate, (b) $\mathrm{MH}$ and $\beta$ crystallinity as a function of the cooling rate, and (c) $\mathrm{MH}$ as a function of the density.

Journal of Polymer Science: Part B: Polymer Physics DOI $10.1002 /$ polb occurring at low cooling rates, to the minimum, observed at high cooling rates, is quite small (30 $\mathrm{MPa}$ ) with respect to the typical $\mathrm{MH}$ drop for other semicrystalline polymers spanning even smaller crystalline contents in the same cooling rate range. ${ }^{44-47}$

Three regions can be distinguished in Figure 6(a): for cooling rates below $1{ }^{\circ} \mathrm{C} / \mathrm{s}$, there is a significant drop in $\mathrm{MH}$ with a remarkable dependence of the mechanical response on the cooling rate; in an intermediate zone (from ca. 1 to ca. $100{ }^{\circ} \mathrm{C} / \mathrm{s}$ ), a mild dependence of $\mathrm{MH}$ on the cooling rate can be observed; and for very high cooling rates $\left(>100{ }^{\circ} \mathrm{C} / \mathrm{s}\right), \mathrm{MH}$ turns out to be almost constant. A sound explanation of this behavior should be drawn from an analysis of the phases formed during solidification.

With reference to Figure 6(a), one should also observe that the reduction of $\mathrm{MH}$ upon the decrease in the content of the crystalline $\alpha$ phase in favor of the amorphous phase is less remarkable than the usual drop observed in semicrystalline polymers. ${ }^{44-47}$

By plotting $\mathrm{MH}$ as a function of the density, one gets the data reported in Figure 6(c). There are two branches clearly visible, which are related to the relative amounts of the phases formed: in the upper part, $\mathrm{MH}$ increases with the density because of the increase in the dense $\beta$-phase content, whereas in the lower part, $\mathrm{MH}$ decreases with increasing density, and this observation should be related to the parallel decrease in the amount of the low-density $\alpha$ phase (in the absence of the $\beta$ phase).

The peculiar dependence of $\mathrm{MH}$ on the cooling rate, shown in Figure 6(a), could then be interpreted on the basis of the density dependence in which densification occurs with an increasing cooling rate, partially compensating for the decrease in the amount of the harder crystalline phase. This leads to an almost constant $\mathrm{MH}$ in the high cooling rate region. As a matter of fact, in this case, the decay of the mechanical performance during the transition from the crystalline $\alpha$ phase to the amorphous phase is probably balanced by the decrease in the volume (densification) occurring as a result of amorphization, that is, the increase in the amorphous content with an increasing cooling rate.

On the other hand, the phase distribution reported in Figure 4 is helpful for explaining the dependence of $\mathrm{MH}$ on the cooling rate, as presented in Figure 6, and the relative difference with respect to the density results. If one com- 
pares the $\mathrm{MH}$ dependence on the cooling rate to the one of the $\beta$ phase [Fig. 6(b)], it becomes evident that the $\mathrm{MH}$ drop-off is basically related to the parallel decrease in the $\beta$-phase content, $\mathrm{MH}$ tending to become almost constant when the $\beta$ phase content is negligible (i.e., $>1{ }^{\circ} \mathrm{C} / \mathrm{s}$ ), although the $\alpha$-phase content keeps on monotonously decreasing. This behavior reveals a high sensitivity of $\mathrm{MH}$ to the denser, highly packed, $\beta$-crystalline phase and a poor sensitivity to the lower density, poorly packed, $\alpha$-crystalline phase, the latter apparently showing an $\mathrm{MH}$ response not very dissimilar from the one of the amorphous phase.

\section{ASSESSMENT OF THE CRYSTALLIZATION KINETICS (KAE APPROACH)}

The study of the crystallization kinetics at atmospheric pressure is the first step toward obtaining good insight into polymer crystallization under processing conditions. ${ }^{31}$ The data available for the description of the crystallization kinetics of the sPS investigated throughout this work cover a very wide range of crystallization conditions. In particular, a consistent set including calorimetric crystallization isotherms and DSC cooling ramps, as well as the final $\alpha$-phase and $\beta$-phase crystallinity contents of quenched samples of a known cooling history, can be considered. As two different phases form, at least two kinetic processes take place simultaneously. The simplest model that one can imagine must parallel two kinetic processes noninteracting and competing for the available molten material. ${ }^{46}$ The kinetic equation adopted here for both processes is the nonisothermal formulation by Nakamura et al. $^{35}$ of the KAE model. ${ }^{32-34}$ The following function, suggested by several authors, ${ }^{48}$ can be adopted for the expression of the time derivative of the volume fraction expectancy, proportional to the fraction of untransformed material times the value of the kinetic constant, in which nucleation and growth rates have been lumped together:

$$
\frac{d \xi_{i}}{d t}=\left(1-\sum_{i} \xi_{i}\right) n_{i} \ln 2\left[\int_{0}^{t} K_{i}(T) d s\right]^{n_{i}-1} K_{i}(T)
$$

where $\xi_{i}$ is the normalized volume fraction of the $i$ th component with respect to the "equilibrium" crystallinity $X_{\text {eq }}$ (see Table 3 ), $n_{i}$ is the Avrami exponent, and $K_{i}$ is the crystallization rate constant.
Table 3. Crystallization Kinetic Parameters According to the KAE Approach

\begin{tabular}{cccccl}
\hline & $K_{\max }\left(\mathrm{s}^{-1}\right)$ & $T_{\max }\left({ }^{\circ} \mathrm{C}\right)$ & $D\left({ }^{\circ} \mathrm{C}\right)$ & $n$ & $X_{\text {eq }}$ \\
\hline$\alpha$ & 0.496 & 198 & 35 & 1 & 0.58 \\
$\beta$ & 0.015 & 237 & 20 & 2.3 & 0.9 \\
\hline
\end{tabular}

As for the dependence of rate constant $K_{i}$ on the temperature, the simplest expression that one can consider is a Gaussian-shaped curve: ${ }^{48}$

$$
K_{i}(T)=K_{\max , i} \exp \left[-\frac{4 \ln 2\left(T-T_{\max , i}\right)^{2}}{D_{i}^{2}}\right]
$$

where $D_{i}, T_{\max , i}$, and $K_{\max , i}$ are the half-width, the temperature at which the maximum of $K_{i}$ is attained, and the maximum value of $K_{i}$ itself, respectively.

The governing equations with reference to two crystalline phases $(\alpha$ and $\beta$ ) are thus the following:

$$
\begin{aligned}
& \frac{d \xi_{\alpha}}{d t}=\left(1-\xi_{\alpha}-\xi_{\beta}\right) n_{\alpha} \ln 2\left[\int_{0}^{t} K_{\alpha}(T) d s\right]^{n_{\alpha}-1} K_{\alpha}(T) \\
& \frac{d \xi_{\beta}}{d t}=\left(1-\xi_{\alpha}-\xi_{\beta}\right) n_{\beta} \ln 2\left[\int_{0}^{t} K_{\beta}(T) d s\right]^{n_{\beta}-1} K_{\beta}(T)
\end{aligned}
$$

It is worth emphasizing that the accessible experimental data were not homogeneous; indeed, they were obtained under different experimental conditions, which consequently led to different data types. In particular, data taken during isothermal tests and constant cooling rate ramps concern enthalpy changes with time, whereas quench experiments supply the final density and crystallinity in quenched samples with a known thermal history. The model parameters for sPS were estimated all together by means of an optimization procedure, implemented in a MATLAB package, by minimization of the quadratic error between the model prediction and data for all the experiments performed. The target of the optimization procedure was the simultaneous agreement of the model predictions with the following:

1. The final crystallinity distribution attained from quenched samples.

Journal of Polymer Science: Part B: Polymer Physics 

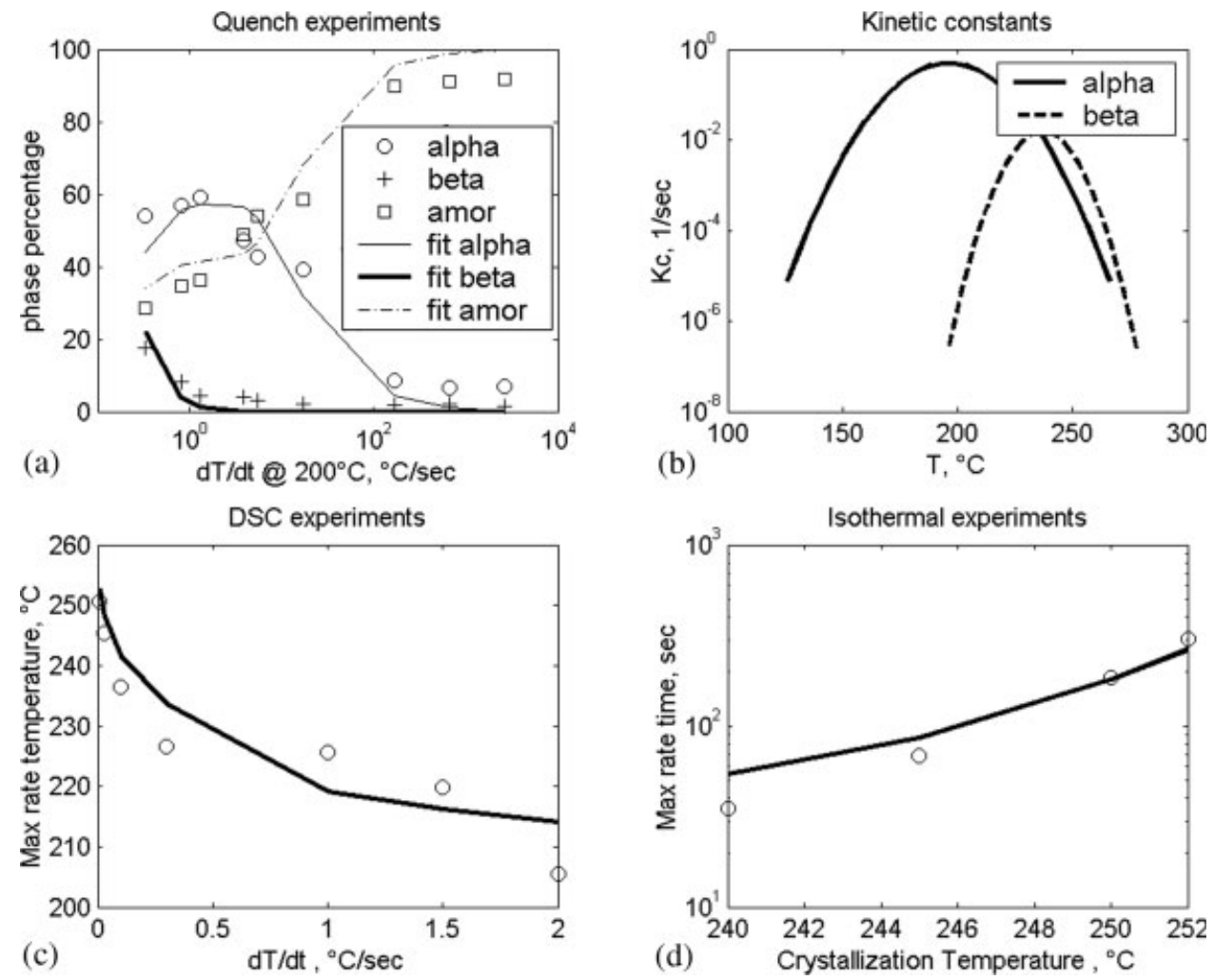

Figure 7. Assessment of the crystallization kinetics by a KAE approach: (a) the phase percentage as a function of the cooling rate (topmost left), (b) the crystallization kinetic constants versus the temperature (topmost right), (c) the DSC cooling ramp maxima versus the cooling rate (bottommost left), and (d) the DSC isothermal run maxima as a function of the temperature (bottommost right).

2. The peak time from isothermal experiments.

3. The peak temperatures from constant cooling rate experiments.

The results of the optimization procedure applied to the available experimental data are shown in Figure 7(a-d), whereas Table 3 reports the optimized parameters. Figure 7(a) shows the phase percentage $(\alpha, \beta$, and amorphous) as a function of the cooling rate together with the predictions obtained according to the aforementioned model; the $\beta$-phase drop is correctly predicted to take place at a cooling rate around $1{ }^{\circ} \mathrm{C} / \mathrm{s}$, whereas the $\alpha$-phase drop is located about 2 decades ahead. Figure 7(b) presents curves of the optimized $\alpha$-phase and $\beta$-phase crystallization kinetic rate constants versus the temperature: the $\beta$-phase crystallization kinetic rate constant lies below the $\alpha$-phase one in the lowtemperature region $\left(<230{ }^{\circ} \mathrm{C}\right)$ and in the high- temperature region $\left(>280{ }^{\circ} \mathrm{C}\right)$, whereas in the intermediate-temperature range, the value of the $\beta$-phase kinetic constant is larger than that of the $\alpha$ phase. This peculiarity determines a sort of phase inversion, which is responsible for the observed density minimum. It depends on an early disappearance of the thermodynamically most favored $\beta$ phase, which forms predominantly in the low cooling rate region, although for extremely low cooling rates, the $\alpha$ phase should prevail. In other words, there is a specific temperature window in which the formation of the $\beta$ phase is favored, approximately positioned between 230 and $280{ }^{\circ} \mathrm{C}$. Figure $7(\mathrm{c}, \mathrm{d})$ reports DSC maxima during cooling ramps and during isothermal experiments, respectively. A reasonably good agreement between the experimental and predicted data is achieved in both cases; it should, however, be recalled that for high cooling rate DSC ramps, the $\alpha$ phase prevails, whereas for low cooling rates, the $\beta$ phase does: 
this may justify the slightly worse fitting results for the DSC cooling run at $2{ }^{\circ} \mathrm{C} / \mathrm{s}$.

\section{CONCLUSIONS}

Conventional DSC analysis (under isothermal and constant cooling rate conditions) was performed for sPS samples to understand the crystallization behavior of this material under mild solidification conditions. This was compared to samples obtained by CCT solidified under more drastic solidification conditions (covering about 5 orders of magnitude in the cooling rate), which emulated conditions normally encountered in polymer processing.

The $\alpha$ phase forms not only during constrained crystallization from the amorphous phase but also when there is constrained mobility, as in the case of solidification from the melt at relatively large cooling rates, which are not large with respect to DSC standards but are as large as those experienced in many polymer processing routes. ${ }^{17}$

The density dependence on the cooling rate exhibits a minimum around $1^{\circ} \mathrm{C} / \mathrm{s}$, which is related to the competition between the densest $\beta$ phase (thermodynamically stable) and the least dense $\alpha$ phase (kinetically favored), as shown by WAXD deconvolution. On the other hand, $\mathrm{MH}$ reduction with the cooling rate may be attributed to the parallel decrease in the $\beta$-phase content. It turns out that the $\alpha$-phase content does not participate in determining the mechanical behavior.

Responsible for this behavior is the peculiar structure of the $\alpha$ phase, ${ }^{49}$ with a density lower than the density of the amorphous phase that can give rise to clathrates as opposed to the $\beta$ phase. The peculiar density relationship of sPS is similar to that of a few other polymers, such as isotactic poly(4-methyl pentene-1), for which amorphization by a pressure increase may be reversibly induced; ${ }^{17,29}$ this behavior has been interpreted through a detailed phase diagram in a recent article. ${ }^{50}$ It would be interesting to verify the expectation that expansion after high cooling rate solidification under pressure would yield the $\alpha$ phase rather than the amorphous phase.

The assessment of the crystallization kinetics in the framework of the KAE approach illustrates the existence of a phase inversion, with a well-defined temperature interval (in the range of $230-280{ }^{\circ} \mathrm{C}$ ), in which $\beta$-phase formation is kinetically favored with respect to the $\alpha$ phase, the latter prevailing in the remaining temperature range. The clearly distinct disappearances of the $\alpha$ and $\beta$ phases with an increasing cooling rate (the former around $1{ }^{\circ} \mathrm{C}$, the latter around $\left.100{ }^{\circ} \mathrm{C} / \mathrm{s}\right)$, together with their respective density differences $\left(\rho_{\alpha}<\rho_{\text {am }}<\rho_{\beta}\right)$, are responsible for the experimentally observed density minimum.

The authors acknowledge the interest of a graduate student, Ing Vitalba Imperiale, who made this work, otherwise not supported by any funding, possible; it was accomplished within a thesis for fulfilling a 5year laurea degree in chemical engineering in the summer session of 2003.

\section{REFERENCES AND NOTES}

1. Yan, R. J.; Ajji, A.; Shinozaki, M. J Mater Sci 1999, 34, 2335-2344.

2. Immirzi, A.; De Candia, F.; Iannelli, P.; Zambelli, A.; Vittoria, V. Makromol Chem Rapid Commun 1988, 9, 761-764.

3. Guerra, G.; Vitagliano, V.; De Rosa, C.; Petraccone, V.; Corradini, P. Macromolecules 1990, 23, 15391544.

4. Evans, A. M.; Kellar, J. C.; Knowles, J.; Galiotis, C.; Carriere, C. J.; Andrews, E. H. Polym Eng Sci 1997, 37, 153-165.

5. Chiu, F.; Shen, K.; Tsai, S. H. Y.; Chen, C. Polym Eng Sci 2001, 41, 881-889.

6. Chiu, F.; Peng, C.; Fu, Q. Polym Eng Sci 2000, 40, 2397-2406.

7. Wu, H. D.; Wu, I. D.; Chang, F. C. Macromolecules 2000, 33, 8915-8917.

8. Olson, B. G.; Prodpran, T.; Jamieson, A. M.; Nazarenko, S. Polymer 2002, 43, 6775-6784.

9. Chatani, Y.; Fujii, Y.; Shimane, Y.; Ijitsu, T. Polym Prepr Jpn (Engl Ed) 1998, 37, 428.

10. Chatani, Y.; Shimane, Y.; Inoue, Y.; Inagaki, T.; Ishioka, T. Polymer 1992, 33, 488-492.

11. Chatani, Y.; Shimane, Y.; Ijitsu, T.; Yukinari, T. Polymer 1993, 34, 1625-1629.

12. Sun, Z.; Morgan, R. J.; Lewis, D. N. Polymer 1992, 33, 660-661.

13. Van Krevelen, D. W. Properties of Polymers; Elsevier: Amsterdam, 1990.

14. Greis, O.; Xu, Y.; Asano, T. Polymer 1989, 30, 590-594.

15. De Rosa, C.; Guerra, G.; Petraccone, V.; Pirozzi, B. Macromolecules 1997, 30, 4147-4152.

16. De Rosa, C.; Rapacciuolo, M.; Guerra, G.; Petraccone, V.; Corradini, P. Polymer 1992, 33, 14231428.

17. Van Hooy-Corstjens, C. S. J.; Holhne, G. W. H.; Rastogi, S. Macromolecules 2005, 38, 1814-1821.

Journal of Polymer Science: Part B: Polymer Physics DOI $10.1002 /$ polb 
18. Barnes, J. D.; McKennia, G. B.; Landes, B. G.; Bubeck, R. A.; Bank, D. Polym Eng Sci 1997, 37, 1480-1484.

19. Rapacciuolo, M.; De Rosa, C.; Guerra, G.; Mensitieri, G.; Apicella, A.; Del Nobile, M. A. J Mater Sci Lett 1991, 10, 1084-1087.

20. De Candia, F.; Guadagno, L.; Vittoria, V. J Macromol Sci Phys 1995, 34, 95-103.

21. Manfredi, C.; De Rosa, C.; Guerra, G.; Rapacciuolo, M.; Auriemma, F.; Corradini, P. Macromol Chem Phys 1995, 196, 2795-2808.

22. De Candia, F.; Filho, A. R.; Vittoria, V. Colloid Polym Sci 1991, 269, 650-654.

23. Auriemma, F.; Petraccone, V.; Dal Poggetto, F.; De Rosa, C.; Guerra, G.; Manfredi, C.; Corradini, P. Macromolecules 1993, 26, 3772-3777.

24. Li, J.; He, J.; Quiang, W.; Hu, X. Polymer 2002, 43, 2489-2494.

25. Filho, A. R.; Vittoria, V. Makromol Chem Rapid Commun 1990, 11, 199-203.

26. Lin, R. H.; Woo, E. M. Polymer 2000, 41, 121131.

27. Bu, W.; Li, Y.; He, J.; Zeng, J. Macromolecules 1999, 32, 7224-7225.

28. Lawrence, S. S.; Shinozaki, D. M. Polym Eng Sci 1997, 37, 1825-1832.

29. Rastogi, S. Proceedings of the Twelfth Annual Meeting of the Polymer Processing Society, Sorrento, Italy, May 27-31, 1996; R. Esposito: Napoli, Italy, 1996; pp 649-650.

30. Handa, Y. P.; Zhang, Z.; Wong, B. Macromolecules 1997, 30, 8499-8504.

31. Brucato, V.; Piccarolo, S.; La Carrubba, V. Chem Eng Sci 2002, 57, 4129-4143.

32. Avrami, M. J. J Chem Phys 1939, 7, 1103-1112.

33. Kolmogoroff, A. N. Isv Akad Nauk Ser Math 1937, 1, 355-359.
34. Evans, U. R. Trans Faraday Soc 1945, 41, 365371.

35. Nakamura, K.; Watanabe, T.; Katayama, K.; Amano, T. J Appl Polym Sci 1972, 16, 1077-1091.

36. Sorrentino, A.; Pantani, R.; Titomanlio, G. J Polym Sci Part B: Polym Phys 2007, 45, 196207.

37. Ding, Z.; Spruiell, J. J Polym Sci Part B: Polym Phys 1996, 34, 2783-2804.

38. Piccarolo, S. J Macromol Sci Phys 1992, 31, 501511.

39. Ciofalo, M.; Di Piazza, I.; Brucato, V. Int J Heat Mass Transfer 1998, 42, 1157-1175.

40. Baltá Calleja, F. J.; Fakirov, S. Microhardness of Polymers; Cambridge University Press: Cambridge, England, 2000.

41. Martorana, A.; Piccarolo, S.; Scichilone, F. Macromol Chem Phys 1997, 198, 597-604.

42. Wunderlich, B. Macromolecular Physics; Academic: New York, 1973; Vol. 1.

43. Strobl, G. The Physics of Polymer (Concepts for Understanding Their Structure and Behaviour); Springer: Munich, 1997.

44. Piccarolo, S.; Brucato, V.; Kiflie, Z. Polym Eng Sci 2000, 40, 1263-1272.

45. La Carrubba, V.; Brucato, V.; Piccarolo, S. Macromol Symp 2002, 180, 43-60.

46. La Carrubba, V.; Brucato, V.; Piccarolo, S. J Polym Sci Part B: Polym Phys 2002, 40, 153-175.

47. La Carrubba, V.; Brucato, V.; Piccarolo, S. Polym Int 2004, 53, 61-68.

48. Ziabicki, A. Fundamentals of Fibre Formation; Wiley: London, 1976.

49. Cartier, L.; Okihara, T.; Lotz, B. Macromolecules 1998, 31, 3303-3310.

50. Rastogi, S.; Vegaa, J. F.; van Rutha, N. J. L.; Terry, A. E. Polymer 2006, 47, 5555-5565. 